TITLE:

\title{
Dual partial dye doping for chromaticity tuning and performance enhancement of white OLEDs
}

\section{$\operatorname{AUTHOR}(\mathrm{S})$ :}

Park, Jongwoon; Suganuma, Naotoshi; Kawakami, Yoichi

\section{CITATION:}

Park, Jongwoon ...[et al]. Dual partial dye doping for chromaticity tuning and performance enhancement of white OLEDs. JOURNAL OF DISPLAY TECHNOLOGY 2008, 4(1): 61-69

\section{ISSUE DATE:}

2008-03

URL:

http://hdl.handle.net/2433/84556

\section{RIGHT:}

C) 2008 IEEE. Personal use of this material is permitted. However, permission to reprint/republish this material for advertising or promotional purposes or for creating new collective works for resale or redistribution to servers or lists, or to reuse any copyrighted component of this work in other works must be obtained from the IEEE. 


\title{
Dual Partial Dye Doping for Chromaticity Tuning and Performance Enhancement of White OLEDs
}

\author{
Jongwoon Park, Naotoshi Suganuma, and Yoichi Kawakami
}

\begin{abstract}
In general, a guest dopant is doped into a single host matrix for white-light emission with two complementary colors. In this work, however, we have fabricated a white organic light-emitting diode (WOLED) based on dual partial dye doping in which a guest dopant is partially doped into two different host emitters; namely, orange-red emitting 4-(dicyanomethylene)-2-methyl-6-( $p$-dimethylaminostyryl)- $4 H$-pyran (DCM) is partially doped into both blue-emitting 4, 4' $4^{\prime}$-bis (2,2'-diphenylvinyl)-1,1'-biphenyl (DPVBi) and green-emitting Tris-(8-hydroxyquinoline) aluminum $\left(\mathrm{Alq}_{3}\right)$. We demonstrate that dual partial dye doping allows us to finely tune the Commission Internationale d'Eclairage (CIE) chromaticity coordinates to the equienergy white point $(x=0.33, y=0.33)$. In addition, it enhances device performance further, compared to WOLEDs based on DCM partially doped into a single host matrix (either DPVBi or $\mathrm{Alq}_{3}$ ). Moreover, the dual partial doping scheme is shown to provide a way of suppressing the self-quenching effect (singlet-singlet annihilation). For a systematic study, we have implemented a comprehensive numerical model and performed simulations of the OLED structure, providing a clear understanding with regard to the underlying physics of OLEDs. We also carry out an investigation of the effects of key design parameters such as the doped layer position and thickness and dye concentration on the electroluminescence property of WOLEDs.
\end{abstract}

Index Terms-Dye doping, electrical simulation, organic lightemitting diode (OLED), white OLED (WOLED).

\section{INTRODUCTION}

W HITE ORGANIC light-emitting diodes (WOLEDs) [1], [2] are essential for many applications including liquid-crystal displays and next generation lightings. For these applications, WOLEDs exhibiting high brightness and efficiency, color stability versus driving voltage, and Commission Internationale d'Eclairage (CIE) chromaticity coordinates close to the equienergy white point $(x=0.33, y=0.33)$ are highly demanded. There have been numerous studies on high-efficiency WOLEDs using a novel material system such as the combination of fluorescent and phosphorescent emitters [3]-[8]. It is however difficult to achieve chromaticity tuning to the equienergy white point with such device configurations because of the formation of singlet (blue emission)

Manuscript received July 9, 2007.

J. Park is with the National Center for Nanoprocess and Equipment, Energy and Applied Optics Team, Korea Institute of Industrial Technology, Gwangju 500-480, Korea (e-mail: pjwup75@kitech.re.kr).

N. Suganuma is with the International Innovation Center, Kyoto University, Kyoto 606-8501, Japan.

Y. Kawakami is with the Department of Electronic Science and Engineering, Kyoto University, Kyoto 615-8510, Japan.

Color versions of one or more of the figures in this paper are available online at http://ieeexplore.ieee.org.

Digital Object Identifier 10.1109/JDT.2007.908362 and triplet (green and red emission) excitons in the ratio of 1:3 due to spin statistics. There have also been intensive researches on white-light generation with two complementary colors using a fluorescent material system [9]-[13]. To obtain white-light emission, red-emitting 5,6,11,12tetraphenylnaphtacene (rubrene) is doped into blue-emitting 4,4'-bis(2,2'-diphenylvinyl)-1,1'-biphenyl (DPVBi) [9] or redemitting \{[2-methyl-6-[2- (2,3,6,7-tetrahydro- $1 \mathrm{H}$, 5H-benzo [ij] quinolizin-9-yl)-ethenyl]-4H-pyran-4-ylidene] propanedinitrile $(\mathrm{DCM} 2)$ is doped into $\mathrm{N}, \mathrm{N}^{\prime}$-diphenyl-N, $\mathrm{N}^{\prime}$-bis $(1-$ naphthyl)-1-1'biphenyl-4,4"diamine ( $\alpha$-NPD) [10]. As the emission peak of rubrene leans more to the green region, the CIE coordinates of those devices are a bit away from the equienergy white point [9], whereas high driving voltage is required for chromaticity tuning to the equienergy white point [10]. Similarly, a current-independent (-balanced) WOLED with DCM2 doped into $\alpha$-NPD has been introduced [11], yet it features very low quantum $(\approx 0.3 \%)$ and power $(0.5 \mathrm{~lm} / \mathrm{W})$ efficiencies. In [12], red-emitting 4-(dicyanomethylene)-2-tert-butyl-6-(1,1,7,7-tetra-methyljulolidin-4-yl-vinyl)-4H-pyran (DCJTB) is doped into Tris-(8-hydroxyquinoline) aluminum $\left(\mathrm{Alq}_{3}\right)$ in WOLED structures with 4,4', $4^{\prime \prime}$-tris(N-3-methylphenyl-N-phenyl-amino)-triphenylamine (m-MTDATA) as a buffer layer and DPVBi as a blue emitter. Due to the enhanced current balance, fairly good device performances were achieved; CIE coordinates $(x=0.3, y=0.31)$ and maximum power efficiency as high as $6.0 \mathrm{~lm} / \mathrm{W}$. However, such a structure requires very high driving voltage. A nondoped OLED structure in which rubrene is inserted between two separated DPVBi layers has been studied lately to avoid difficulty in doping control process [13]. It shows the CIE coordinates $(x=0.33, y=0.32)$, external quantum efficiency $\left(\eta_{\text {ext }}\right)$ of $1.9 \%$, and luminance of $2,234 \mathrm{~cd} / \mathrm{m}^{2}$ at $60 \mathrm{~mA} / \mathrm{cm}^{2}$.

Those fluorescent WOLEDs discussed above have a common feature; a guest dopant is doped into a single host matrix. In this work, however, we study a novel fluorescent WOLED configuration based on dual partial dye doping. The main peculiarity of this structure is that a guest dopant (4-(dicyanomethylene)-2-methyl-6-( $p$-dimethylaminostyryl)- $4 H$-pyran (DCM)) is partially doped into two different host emitters (DPVBi and $\mathrm{Alq}_{3}$ ) to enhance device efficiency and finely tune the CIE coordinates to the equienergy white point. We first investigate the carrier transport property of the OLED structure by solving Poisson's equation, time-dependent drift-diffusion equations, and singlet exciton rate equation iteratively. We then analyze the electroluminescence (EL) properties of the WOLED structure; in particular, the influences of varying the doped layer position and thickness and dye concentration are examined. Through a performance comparison among different 
WOLEDs, we finally demonstrate that the dual partial doping scheme indeed enhances the overall device performance and provides a method of depressing singlet-singlet annihilation (self-quenching).

This paper is organized as follows. In Section II, the numerical model for the simulation of OLED structures is briefly described. Simulation and measurement results, e.g., the carrier and exciton distributions inside OLED devices, EL spectra, CIE coordinates, and device efficiencies, are presented and analyzed in Section III. Finally, a conclusion is drawn in Section IV.

\section{MODEL}

With attempt to gain some insight into the underlying physics and complex behaviors of OLEDs in response to a variety of design parameters such as the doped layer position and thickness, we have implemented a 1D numerical model consisting of Poisson's equation, time-dependent drift-diffusion equations, and singlet exciton rate equation [14]-[18]. In this model, the current injection into organic materials, the hopping transport of carriers, the locally generated excited states (excitons), the radiative decay of excitons, as well as the interactions among the electric field, the carrier mobility, and the current injection are all included.

Poisson's equation governing the time- and space-dependent electric field can be expressed by the standard difference approximation as

$$
\begin{aligned}
E\left(x+\frac{\Delta x}{2}, t\right)=E\left(x-\frac{\Delta x}{2}, t\right) & +\Delta x \frac{q}{\varepsilon}\{p(x, t)-n(x, t) \\
& \left.+N_{D}(x)-N_{A}(x)\right\}
\end{aligned}
$$

where the variable $E(x, t)(\mathrm{V} / \mathrm{cm})$ denotes the electric field, $\Delta x$ $(=0.2 \mathrm{~nm})$ the cell width discretized in space, $n(x, t)\left(\mathrm{cm}^{-3}\right)$ and $p(x, t)\left(\mathrm{cm}^{-3}\right)$ the electron and hole densities, respectively, and $N_{D}(x)\left(\mathrm{cm}^{-3}\right)$ and $N_{A}(x)\left(\mathrm{cm}^{-3}\right)$ the donor and acceptor impurity concentrations, respectively. The spatial electric field profile and carrier (electron and hole) density distribution can be calculated at each time step by solving (1) along with the following drift-diffusion equation written as

$$
\begin{aligned}
& n(x, t+\Delta t) \\
& =n(x, t)+\Delta t\left\{\frac{1}{q} \frac{J_{n}\left(x+\frac{\Delta x}{2}, t\right)-J_{n}\left(x-\frac{\Delta x}{2}, t\right)}{\Delta x}\right. \\
& \quad-r(x, t) n(x, t) p(x, t)\} \\
& p(x, t+\Delta t) \\
& =p(x, t)-\Delta t\left\{\frac{1}{q} \frac{J_{p}\left(x+\frac{\Delta x}{2}, t\right)-J_{p}\left(x-\frac{\Delta x}{2}, t\right)}{\Delta x}\right. \\
& +r(x, t) n(x, t) p(x, t)\}
\end{aligned}
$$

with the recombination rate $r$ in the Langevin form $r(x, t)=$ $q\left(\mu_{n}(x, t)+\mu_{p}(x, t)\right) / \varepsilon$ [17]. The current densities appearing in (2) are given as

$$
\begin{aligned}
J_{n}\left(x+\frac{\Delta x}{2}, t\right)= & q \mu_{n}\left(x+\frac{\Delta x}{2}, t\right) \frac{n(x, t)+n(x+\Delta x, t)}{2} \\
& \times E\left(x+\frac{\Delta x}{2}, t\right)+k T \mu_{n}(x+\Delta x 2, t) \\
& \times \frac{n(x+\Delta x, t)-n(x, t)}{\Delta x} \\
J_{p}\left(x+\frac{\Delta x}{2}, t\right)= & q \mu_{p}\left(x+\frac{\Delta x}{2}, t\right) \frac{p(x, t)+p(x+\Delta x, t)}{2} \\
& \times E\left(x+\frac{\Delta x}{2}, t\right)-k T \mu_{p}\left(x+\frac{\Delta x}{2}, t\right) \\
& \times \frac{p(x+\Delta x, t)-p(x, t)}{\Delta x}
\end{aligned}
$$

where the variable $\Delta t(=1 \mathrm{ps})$ represents the time step, $\mu_{n}(x, t)\left(\mathrm{cm}^{2} / \mathrm{V} \cdot \mathrm{s}\right)$ the electron mobility, $\mu_{p}(x, t)\left(\mathrm{cm}^{2} / \mathrm{V} \cdot \mathrm{s}\right)$ the hole mobility, $k(\mathrm{~J} / \mathrm{K})$ the Boltzmann constant, and $T(\mathrm{~K})$ the temperature $(=300 \mathrm{~K})$. We have employed the Poole-Frenkel like carrier mobility expressed as [19], [20]

$$
\mu(E(x, t))=\mu_{0} \exp \left(\sqrt{\frac{E(x, t)}{E_{0}}}\right)
$$

with $\mu_{0}\left(\mathrm{~cm}^{2} / \mathrm{V} \cdot \mathrm{s}\right)$ defined as the zero-field mobility and $E_{0}$ $\left(\mathrm{V} \cdot \mathrm{cm}^{-1}\right)$ the characteristic field.

After solving (1) and (2), the singlet exciton density distribution can be obtained by updating the following rate equation [14], [16], [21]:

$$
\begin{aligned}
S(x, t+\Delta t) & \\
= & S(x, t)+\Delta t \\
\times & \left\{\frac{1}{4} r(x, t) n(x, t) p(x, t)-\frac{S(x, t)}{\tau_{s}}-Q(x) \frac{S(x, t)}{\tau_{q}}\right. \\
& \left.\quad+D_{s} \frac{S(x+\Delta x, t)-2 S(x, t)+S(x-\Delta x, t)}{\Delta x^{2}}\right\}
\end{aligned}
$$

where

$$
Q(x)=\exp \left(-\frac{x}{L}\right)+\exp \left(-\frac{L-x}{L}\right)
$$

with $L(\mathrm{~cm})$ denoting the device length. The first term inside the bracket in the right-hand side of (5) represents the singlet exciton generation, the second term the photon generation, the third term the quenching effects at the electrodes, and the last term the exciton diffusion. In (5), $D_{s}$ denotes the diffusion constant written as $D_{s}=l_{d}^{2} / \tau_{s}$ with $l_{d}(\mathrm{~cm})$ defined as the diffusion length, and $\tau_{s}$ (s) and $\tau_{q}$ (s) the exciton lifetime (=10 ns) and the reduced lifetime (= $1 \mathrm{~ns}$ ) by the exciton quenching at the electrodes, respectively. More detailed description of the model 


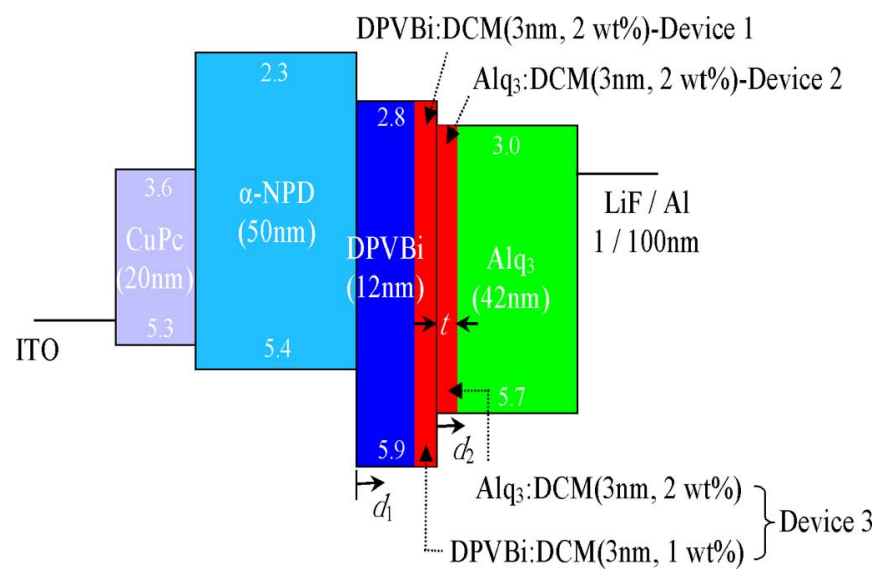

Fig. 1. Schematic view of the layer structure and energy level of WOLEDs.

including boundary conditions for the electric field and current injection into the organic materials is provided in [21].

The model we have implemented in this paper is relatively simple as we do not intend to make a quantitative comparison with experimental results, but to make full use of such a simple model for capturing the underlying physics of OLEDs that may help one analyze their complex behaviors observed experimentally and thus optimize the device configuration. To take the dopant effect into account, one has to include Förster energy transfer and singlet-singlet annihilation in the singlet exciton rate equations of host and dopant [22], together with carrier trapping at the dopant sites in the drift-diffusion equations.

\section{RESULTS AND DISCUSSION}

As illustrated in Fig. 1, the WOLED structures that we have fabricated consist essentially of a 110-nm-thick indium-tin-oxide (ITO) pre-coated on a glass substrate, 20-nm-thick copper phthalocyanine (CuPc), 50-nm-thick $\alpha$-NPD, 15-nm-thick DPVBi, 45-nm-thick $\mathrm{Alq}_{3}$, 1-nm-thick lithium fluoride $(\mathrm{LiF})$, and 100-nm-thick aluminum $(\mathrm{Al}) \mathrm{de}$ posited sequentially at a pressure around $3.0 \times 10^{-4} \mathrm{~Pa}$. In Device 1, the 3-nm-thick DCM-doped (2 wt\%) layer is positioned inside the 15-nm-thick DPVBi layer. By shifting the DCM layer (the doping area) from the $\alpha$-NPD/DPVBi interface to the DPVBi $/ \mathrm{Alq}_{3}$ interface (namely, by increasing $d_{1}$ from zero up to $12 \mathrm{~nm}$ by $3 \mathrm{~nm}$ ), we seek for the place where DCM can be doped for white-light emission by way of experiment in conjunction with simulation that provides a clear understanding on the device physics. For the same purpose, the 3-nm-thick DCM-doped (2 wt \%) layer has been shifted inside the $\mathrm{Alq}_{3}$ layer (Device 2) from the DPVBi $/ \mathrm{Alq}_{3}$ interface to the $\mathrm{Al}$ electrode (i.e., $d_{2}$ is increased). Unlike Device 1 and Device 2 where DCM is partially doped into a single host emitter (DPVBi or $\mathrm{Alq}_{3}$ ), it is doped partially into both DPVBi and $\mathrm{Alq}_{3}$ at the DPVBi$/ \mathrm{Alq}_{3}$ interface in Device 3 (dual partial dye doping: $1 \mathrm{wt} \%$ DCM into DPVBi and $2 \mathrm{wt} \%$ into $\mathrm{Alq}_{3}$ ). Through a performance comparison with Device 1 and Device 2 , we also investigate the effect of such dual partial dye doping on chromaticity tuning and device performance. The EL property was measured using a SourceMeter (2400, Keithley Instrument, Inc.), multi-function optical meter (1835C, Newport Corp.), and photonic multi-channel analyzer (PMA-11

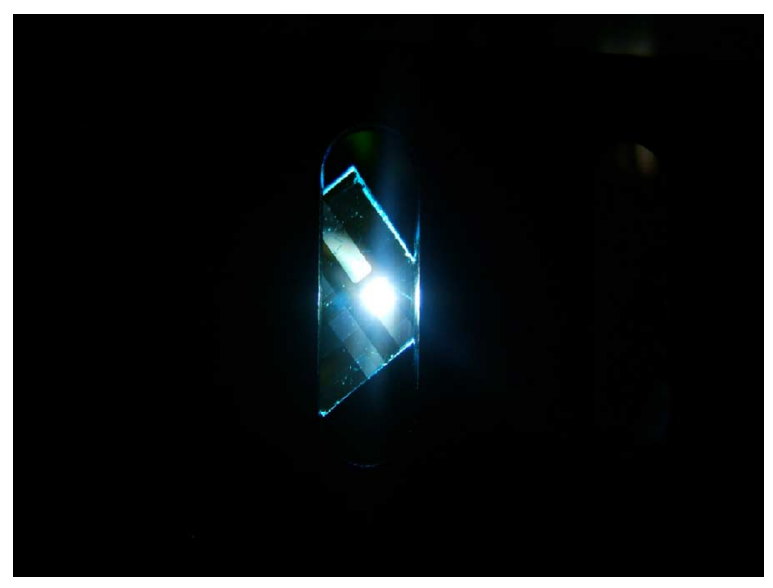

Fig. 2. Snapshot of EL emission from Device 3 at $11 \mathrm{~V}$.

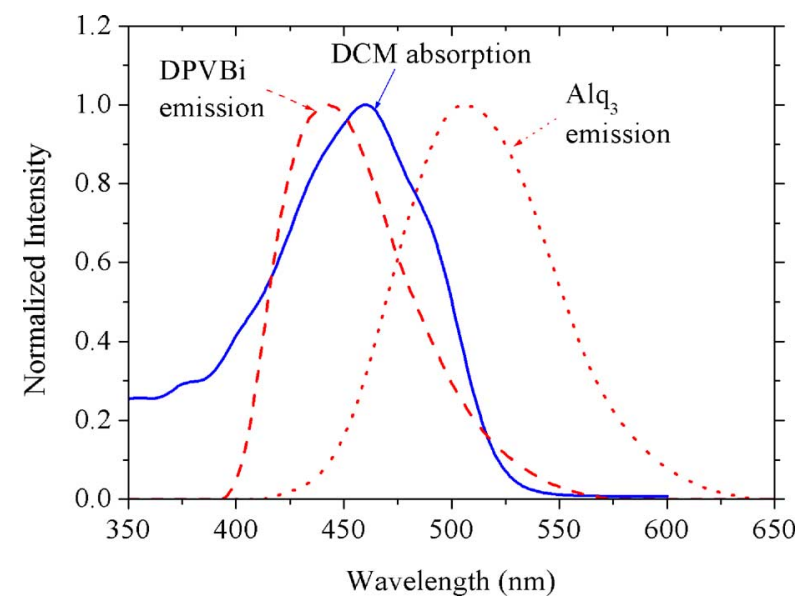

Fig. 3. Absorption spectrum of DCM and photoluminescence spectra of DPVBi and $\mathrm{Alq}_{3}$.

C7473-36, Hamamatsu Photonics K.K.). All the measurements were carried out at room temperature without any encapsulation. Fig. 2 displays the snapshot of white-light emission from Device 3 under the bias voltage of 11 V. Shown in Fig. 3 are the absorption spectrum of DCM and the photoluminescence spectra of DPVBi and $\mathrm{Alq}_{3}$ diluted with chloroform. We can see a good overlap between the absorption and emission spectra, which enables DCM doping into both DPVBi and $\mathrm{Alq}_{3}$ (i.e., a necessary condition for dual partial dye doping) since efficiency energy transfer from the hosts to the guest is expected.

To facilitate the simulation, we have divided the entire device into $M(=550)$ subsections (cells) so that the spatial variation of the electric field and charge carriers is accounted for. The cell width $(\Delta x=0.2 \mathrm{~nm})$ is chosen to be comparable to the distance between organic molecules. The barrier height for holes and electrons is assumed to be $0.4 \mathrm{eV}$ and $0.3 \mathrm{eV}$, respectively, at $300 \mathrm{~K}$. The energy level offsets in the lowest unoccupied molecular orbital (LUMO) and the highest occupied molecular orbital (HOMO) at the organic/organic interfaces (Fig. 1) are extracted from the open literature [12]-[14]. Table I lists the parameter values related to exciton diffusion [13] and carrier mobilities [14], which are typical for such a device with the material system chosen. 


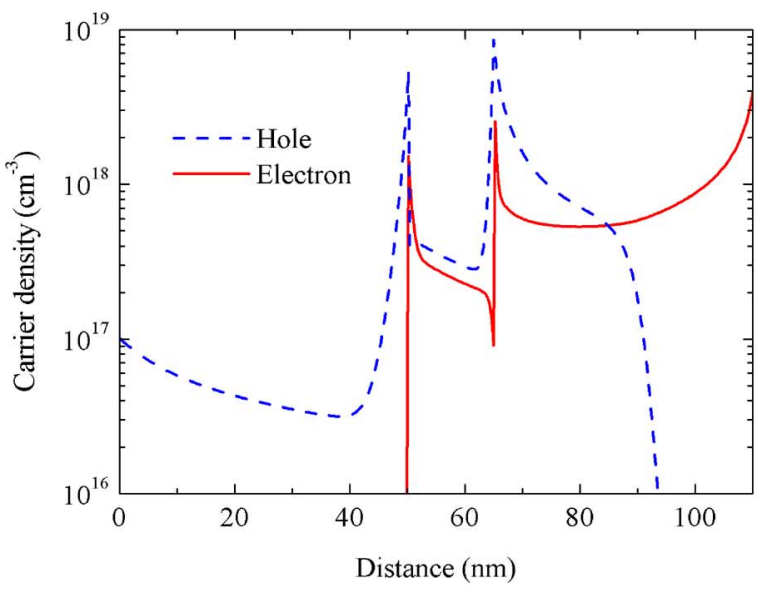

(a)

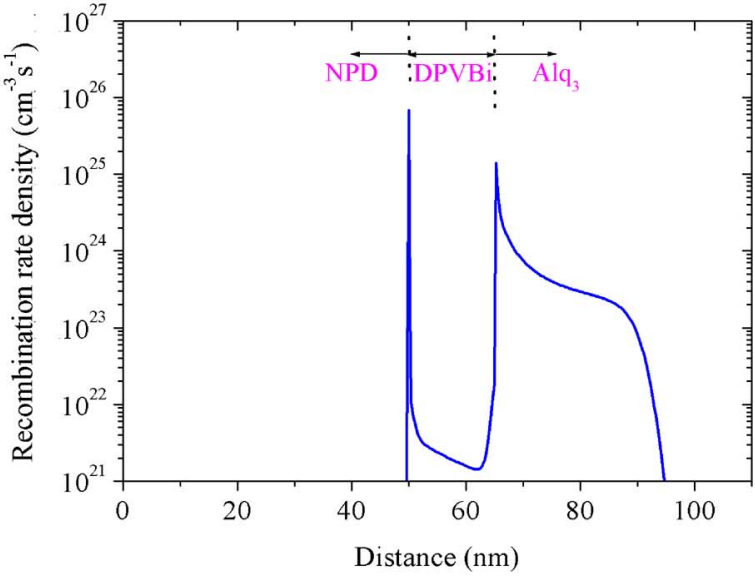

(b)

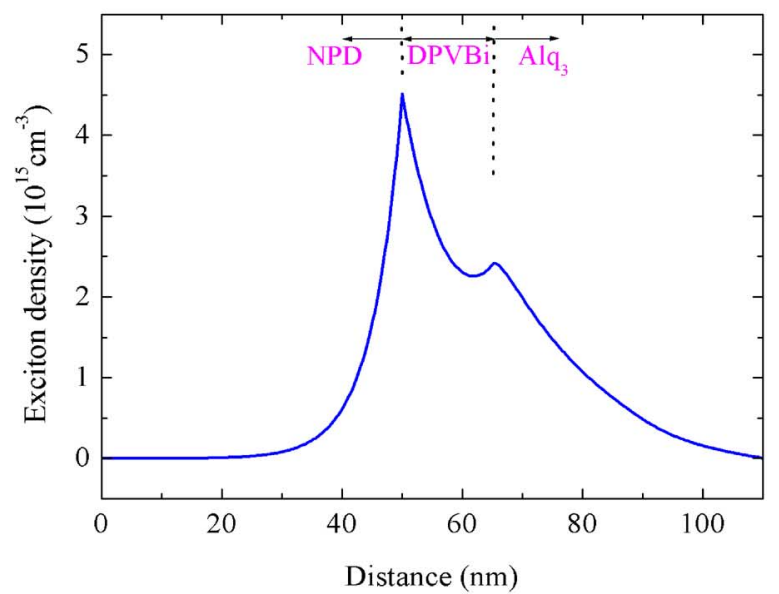

(c)

Fig. 4. Simulation results of (a) carrier density, (b) recombination rate density, and (c) exciton density distributions inside the WOLED structure at $10 \mathrm{~V}$ with the DCM dopant excluded.

TABLE I

MATERIAL PARAMETER VALUES

\begin{tabular}{llllll}
\hline Layer & $\mu_{o, n}\left(\mathrm{~cm}^{2} / \mathrm{Vs}\right)$ & $\mu_{o, p}\left(\mathrm{~cm}^{2} / \mathrm{Vs}\right)$ & $E_{o, n}(\mathrm{~V} / \mathrm{cm})$ & $E_{o, p}(\mathrm{~V} / \mathrm{cm})$ & $l_{d}(\mathrm{~nm})$ \\
\hline$\alpha$-NPD & $1 \times 10^{-8}$ & $4.3 \times 10^{-4}$ & $1 \times 10^{5}$ & $1 \times 10^{6}$ & 5 \\
DPVBi & $2 \times 10^{-8}$ & $2 \times 10^{-8}$ & $11.8 \times 10^{3}$ & $11.8 \times 10^{3}$ & 9 \\
Alq $_{3}$ & $1.2 \times 10^{-6}$ & $0.12 \times 10^{-6}$ & $1959 \times 10^{3}$ & $1959 \times 10^{3}$ & 10 \\
\hline
\end{tabular}

\section{A. Simulation Results}

By solving those governing equations (1), (2), and (5) in a self-consistent manner, we can calculate the spatial variation of the carrier density, recombination rate density, and exciton density inside the OLED device. Fig. 4 shows the simulation results of their spatial variation obtained under the bias voltage of $10 \mathrm{~V}$ without DCM dopant involved. As seen in Fig. 4(a), most of carriers are piled up at the organic/organic interfaces due to the energy level offset and mobility discontinuity [21]. Consequently, electron-hole recombination (singlet exciton formation) arises dominantly near the NPD/DPVBi and DPVBi/Alq ${ }_{3}$ interfaces as evident in Fig. 4(b). Due most likely to larger energy level offset (Fig. 1), more pronounced electron-hole recombination takes place at the NPD/DPVBi interface. Those simulation results will provide a clear understanding of experimental results discussed below. Interestingly, no significant recombination occurs in the middle of the DPVBi layer even though the carrier mobility of DPVBi is much lower than that of the other host materials. However, "seed" excitons formed initially at those interfaces are diffused and distributed in such a manner as seen in Fig. 4(c). One can clearly see that excitons are confined to some extent inside the emitting (DPVBi) layer with low carrier mobility. This simulation result gives us an important hint that one can possibly suppress singlet-singlet annihilation with the dual partial dye doping scheme, since singlet excitons generated from each layer could be decoupled or rather confined in the respective layer. We shall return to this point later with experimental data.

\section{B. Experiment Results}

To validate qualitatively the simulation results, we have first fabricated the OLED device (Fig. 1) without any DCM doping and measured the EL spectrum presented in Fig. 5. As most of seed excitons are formed at the NPD/DPVBi interface [Fig. 4(c)], the emission peak is observed to be in the blue region $(450 \mathrm{~nm})$. Although blue emission from DPVBi is dominant, one can clearly see relatively weak green emission from $\mathrm{Alq}_{3}$, a phenomenon expected from the simulation result of exciton distribution in Fig. 4(c); a large number of excitons 


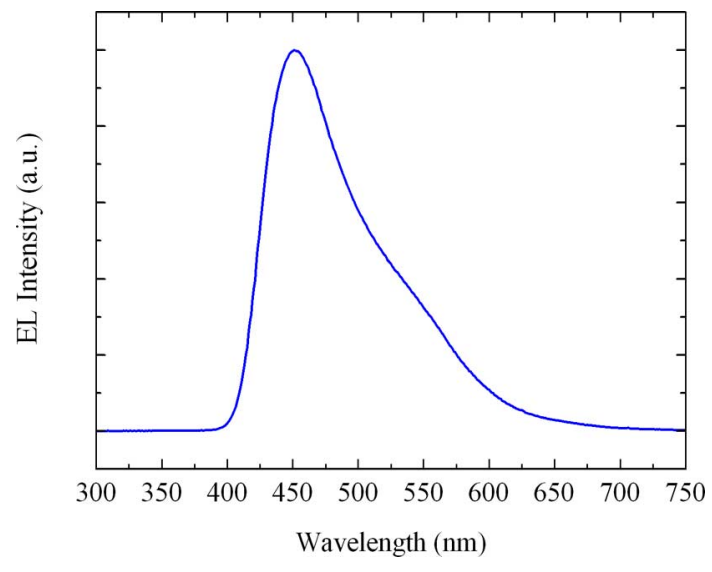

Fig. 5. EL spectra of the OLED without any DCM doping measured at $10 \mathrm{~V}$.

diffuse into the $\mathrm{Alq}_{3}$ layer, thereby making green emission perceptible.

We have shifted the 3-nm-thick DCM-doped layer inside the 15-nm-thick DPVBi layer (Device 1) from the NPD/DPVBi interface toward the DPVBi $/ \mathrm{Alq}_{3}$ interface (i.e., increased $d_{1}$ by $3 \mathrm{~nm}$ ) in order to locate a spot where DCM can be doped for white-light emission. Provided in Fig. 6 are the measured EL spectra and corresponding CIE coordinates of Device 1 varying depending on the DCM layer position $\left(d_{1}\right)$. When $d_{1}=0$ (i.e., 2 wt $\%$ DCM is partially $(3 \mathrm{~nm})$ doped into the DPVBi layer at the NPD/DPVBi interface), DCM emission is dominant, though the blue-emitting undoped area (i.e., 12-nm-thick DPVBi) is larger than the DCM-doped region (3-nm-thick). It is attributed to the fact that most efficient energy transfer from host excitons to guest ones happens near the NPD/DPVBi interface, as expected from the simulation result in Fig. 4(c). However, blue emission is getting pronounced with increasing $d_{1}$, since the number of host excitons that contribute to energy transfer to guest excitons is decreased. Consequently, the CIE coordinates approach the equienergy white point as evident in Fig. 6(b). From the results presented in Fig. 6, therefore, one needs to dope DCM near the $\mathrm{DPVBi} / \mathrm{Alq}_{3}$ interface (into the DPVBi layer) for white-light emission.

To find out the optimal DCM concentration required for white-light generation, we have increased the DCM concentration up to $4 \mathrm{wt} \%$ in Device $1\left(d_{1}=12 \mathrm{~nm}\right)$ and measured the EL spectra shown in Fig. 7. With increasing DCM concentration, yellow-red emission is getting strong, the CIE coordinates by which become apart from the equienergy white point. Moreover, singlet-singlet annihilation (self-quenching) induced by high DCM concentration ( $>2 \mathrm{wt} \%$ ) may degrade the overall device performance [12], [23]. We shall return to this point later with more experimental results. For those reasons, we restrict the DCM concentration within the range $1 \%-2 \%$ by weight in this work.

The color stability versus driving voltage is one of key figures of merit for WOLEDs. Shown in Fig. 8 are the EL spectra and corresponding CIE coordinates of device $1\left(d_{1}=12 \mathrm{~nm}\right)$ measured under different bias voltages. Good color stability has been observed, though it may depend sensitively on the DCM concentration and the doped layer thickness.

In a similar manner, we have also shifted the 3-nm-thick $\operatorname{DCM}(2 \mathrm{wt} \%)$ layer inside the $\mathrm{Alq}_{3}$ layer from the $\mathrm{DPVBi} / \mathrm{Alq}_{3}$

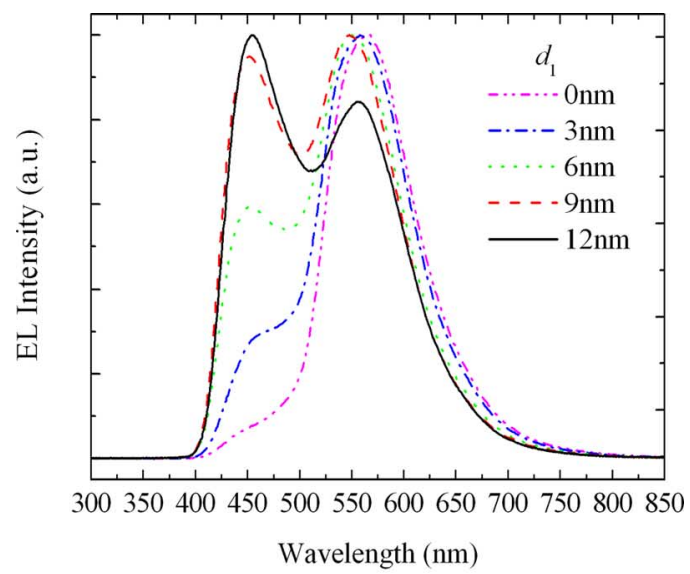

(a)

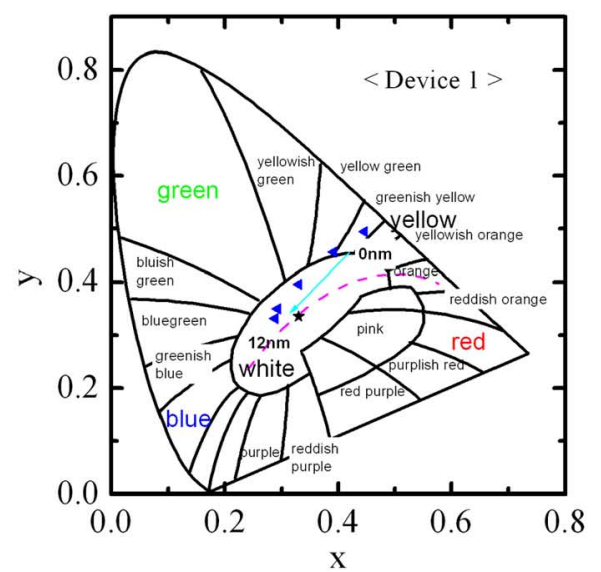

(b)

Fig. 6. (a) EL spectra measured at $10 \mathrm{~V}$ and (b) associated CIE coordinates of Device 1 varying depending on the DCM (2 wt $\%)$ layer position $\left(d_{1}\right)$ inside the DPVBi layer. The point where the symbol $\square$ is marked is corresponding to the equienergy white point. The dash line represents black body curve.

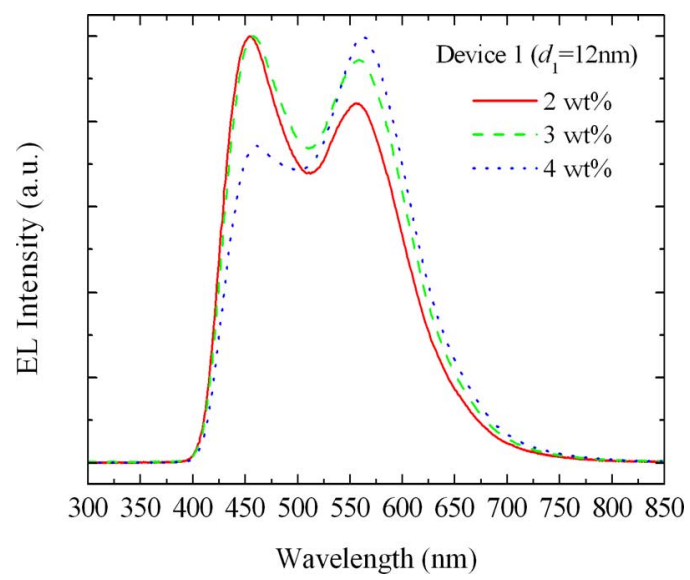

Fig. 7. EL spectra of device 1 for different DCM concentrations at $10 \mathrm{~V}$.

interface toward the $\mathrm{Al}$ electrode (i.e., increased $d_{2}$ by $4 \mathrm{~nm}$ ) in Device 2 and measured the EL spectra and associated CIE coordinates presented in Fig. 9. As expected from the simulation result in Fig. 4(c), DCM emission is getting weakened with increasing $d_{2}$ because less host excitons are available for energy transfer to guest excitons. As a result, the CIE coordinates go away from the equienergy white point. Therefore, it is desirable 


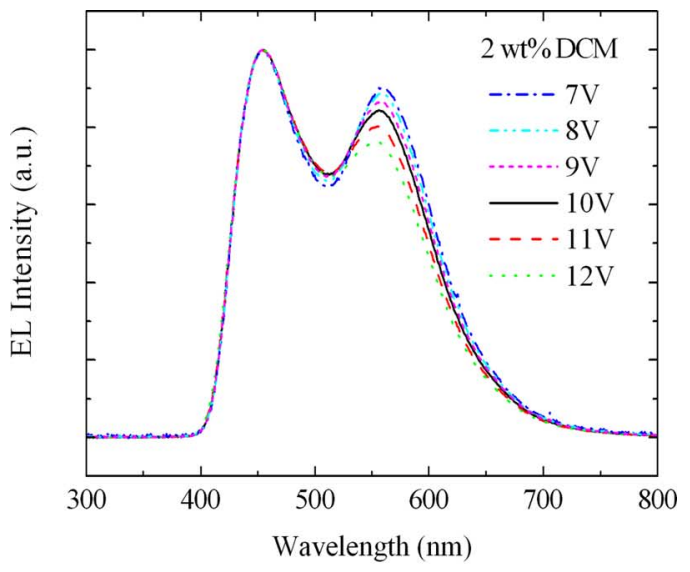

(a)

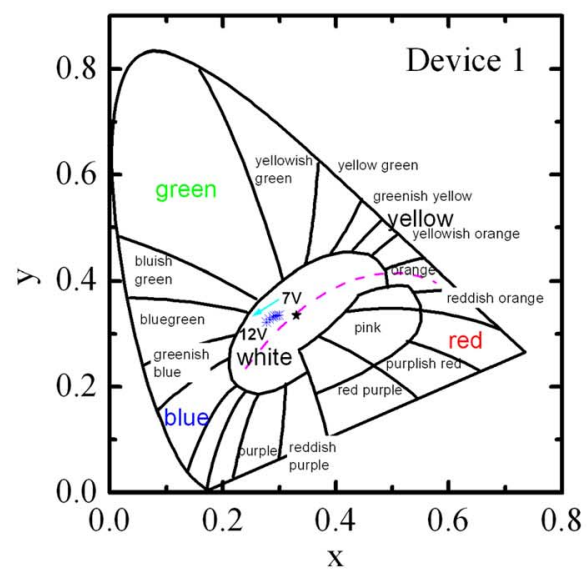

(b)

Fig. 8. (a) EL spectra and (b) corresponding CIE coordinates of device 1 measured under different bias voltages when $d_{1}=12 \mathrm{~nm}$.

to dope DCM near the DPVBi/Alq 3 interface (into the $\mathrm{Alq}_{3}$ layer) for white-light emission close to the equienergy white point.

Furthermore, we have increased the DCM layer thickness $(t)$ of Device $2\left(d_{2}=0\right)$ and observed the EL property shown in Fig. 10. It appears that blue emission becomes reduced with increasing $t$ and hence the CIE coordinates go away from the equi-energy white point, due possibly to an increase in carrier trapping by the guest dopant populated in wider area. Namely, such carrier trapping in the $\mathrm{Alq}_{3}$ layer prohibits carriers from being transported to the NPD/DPVBi interface where strong blue emission occurs. As such, the DCM layer thickness needs to be limited to $6 \mathrm{~nm}$ for the DCM concentration of $2 \%$ by weight.

In brief, for white-light emission, it is desirable to dope DCM near the DPVBi $/ \mathrm{Alq}_{3}$ interface (rather than the NPD/DPVBi interface) into either the DPVBi or $\mathrm{Alq}_{3}$ layer within the limit of the concentration of $2 \mathrm{wt} \%$ and the doped layer thickness of $6 \mathrm{~nm}$. For a comparative study in the subsequent subsection, hence, we have chosen $d_{1}$ to be $12 \mathrm{~nm}$ in Device $1, d_{2}$ to be $0 \mathrm{~nm}$ in Device 2, and $t$ to be $3 \mathrm{~nm}$ in both; namely, ITO $/ \operatorname{CuPc}(20 \mathrm{~nm}) / \alpha-$ $\operatorname{NPD}(50 \mathrm{~nm}) / \operatorname{DPVBi}(12 \mathrm{~nm}) / \mathrm{DPVBi}: \operatorname{DCM}(3 \mathrm{~nm}) / \mathrm{Alq}_{3}$ $(45 \mathrm{~nm}) / \operatorname{LiF}(1 \mathrm{~nm}) / A l(100 \mathrm{~nm}) \quad($ Device 1$)$ and $\mathrm{ITO} / \mathrm{CuPc}(20 \mathrm{~nm}) / \alpha-\mathrm{NPD}(50 \mathrm{~nm}) / \operatorname{DPVBi}(15 \mathrm{~nm}) / \mathrm{Alq}_{3}$ :

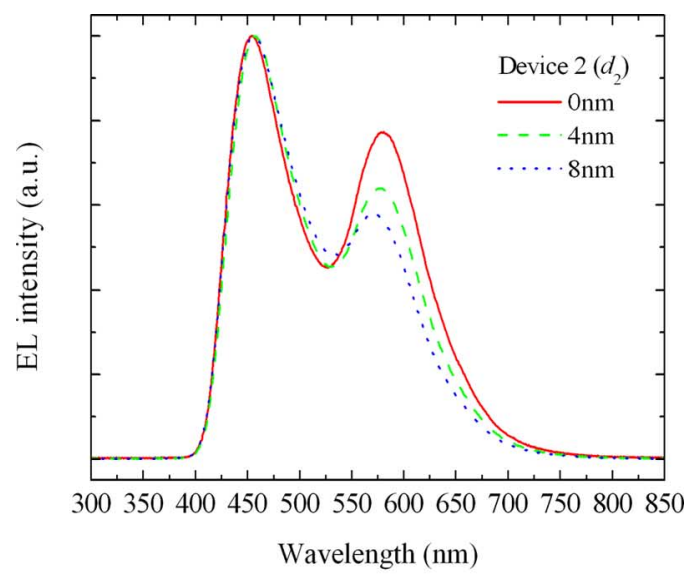

(a)

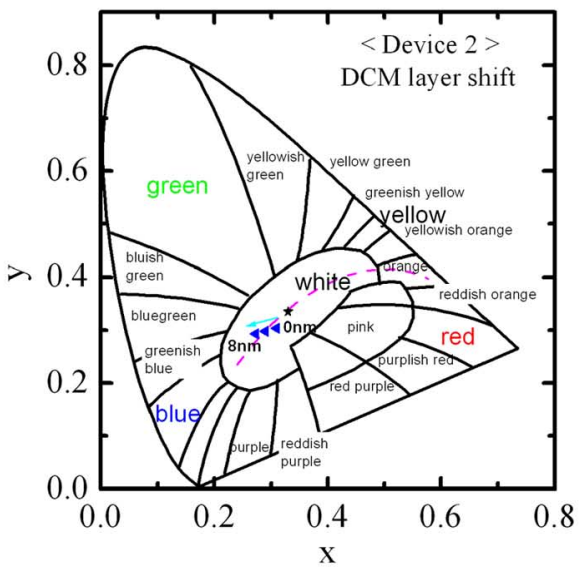

(b)

Fig. 9. (a) EL spectra measured at $10 \mathrm{~V}$ and (b) associated CIE coordinates of Device 2 varying depending on the DCM (2 wt $\%)$ layer position $\left(d_{2}\right)$ inside the $\mathrm{Alq}_{3}$ layer.

$\operatorname{DCM}(3 \mathrm{~nm}) / \mathrm{Alq}_{3}(42 \mathrm{~nm}) / \operatorname{LiF}(1 \mathrm{~nm}) / \operatorname{Al}(100 \mathrm{~nm})$ (Device 2) (also see Fig. 1).

\section{Comparison Among WOLEDs}

In this subsection, we make a performance comparison among those WOLEDs based on single partial dye doping (Devices 1 and 2) and WOLED based on dual partial dye doping (Device 3 illustrated in Fig. 1).

Shown in Fig. 11 are the EL spectra and associated CIE coordinates of those WOLEDs measured at $10 \mathrm{~V}$. It is observed that the DCM emission peak is nearly 24-nm red-shifted when the DCM layer is shifted from DPVBi to $\mathrm{Alq}_{3}$ (Device $1 \rightarrow$ Device 2), which may be induced by the different interaction between host and guest dipoles. It is a desired feature because the CIE coordinates approach the equienergy white point $(x=0.33, y=$ $0.33)$ thanks to the redder component in the spectrum. The CIE coordinates of Device 1 and Device 2 are measured to be $(x=$ $0.29, y=0.33)$ and $(x=0.31, y=0.303)$, respectively, both of which are still far from the equienergy white point. However, the dual partial doping scheme applied to Device 3 enables chromaticity tuning to the equienergy white point, as demonstrated in Fig. 11(b) where the CIE coordinates of Device 3 are marked at $(x=0.331, y=0.337)$. It is feasible because the overlap between two emission spectra (one from DCM-doped DPVBi and 


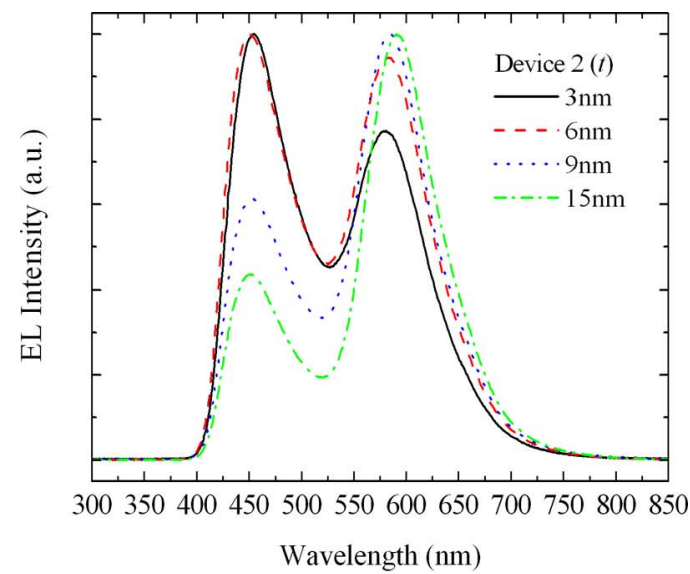

(a)

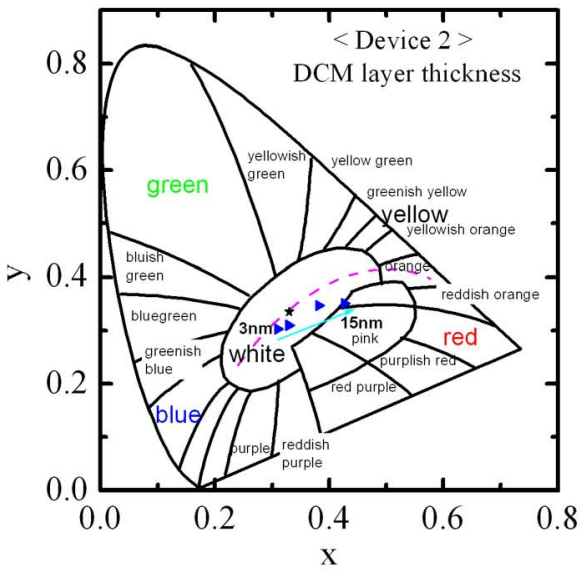

(b)

Fig. 10. (a) EL spectra measured at $10 \mathrm{~V}$ and (b) corresponding CIE coordinates of Device 2 for different DCM layer thicknesses $(t)$ inside the $\mathrm{Alq}_{3}$ layer when $d_{2}=0$.

the other from DCM-doped $\mathrm{Alq}_{3}$ ) broadens the DCM emission bandwidth (Fig. 11(a)) ranging from green to red.

We have also measured the current density and luminance as a function of bias voltage and presented the data in Fig. 12. It is found that Device 3 exhibits the highest luminance $(2,575$ $\mathrm{cd} / \mathrm{m}^{2}$ at $\left.10 \mathrm{~V}\right)$, followed by Device $2\left(2,386 \mathrm{~cd} / \mathrm{m}^{2}\right.$ at $\left.10 \mathrm{~V}\right)$ and Device $1\left(2,140 \mathrm{~cd} / \mathrm{m}^{2}\right.$ at $\left.10 \mathrm{~V}\right)$. The external quantum efficiency $\left(\eta_{\text {ext }}\right)$ of Device 3 is measured to be almost the same as that $(1.96 \%)$ of Device 2 , which is comparable with the result in [13]. On the other hand, Device 1 provides the lowest $\eta_{\text {ext }}$ (as low as 1.48\%). One can also see from Fig. 13 that Device 3 features the highest power efficiency $(3.44 \mathrm{~lm} / \mathrm{W})$, followed by Device $2(3.16 \mathrm{~lm} / \mathrm{W})$ and Device $1(2.58 \mathrm{~lm} / \mathrm{W})$. Those measured values are summarized in Table II. We can conclude from these that dual partial dye doping is highly desired not only for chromaticity tuning but for performance enhancement.

It would be possible to achieve chromaticity tuning to the equienergy white point by increasing the DCM concentration further $(>2 \mathrm{wt} \%)$ in Device 1 and Device 2 without employing the dual partial doping scheme. As mentioned earlier, however, singlet-singlet annihilation would degrade the device performance. To confirm it, we have increased the DCM concentration up to $3 \mathrm{wt} \%$ in Device 1 and Device 2 and then made a performance comparison with Device 3 that has the same overall

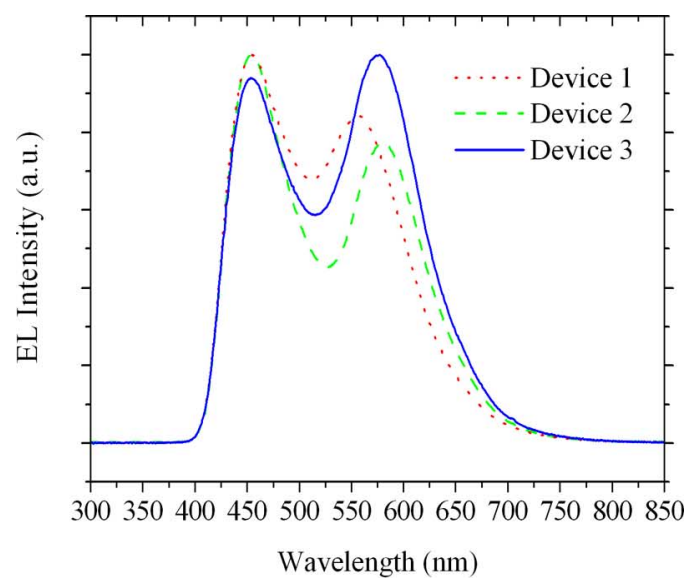

(a)

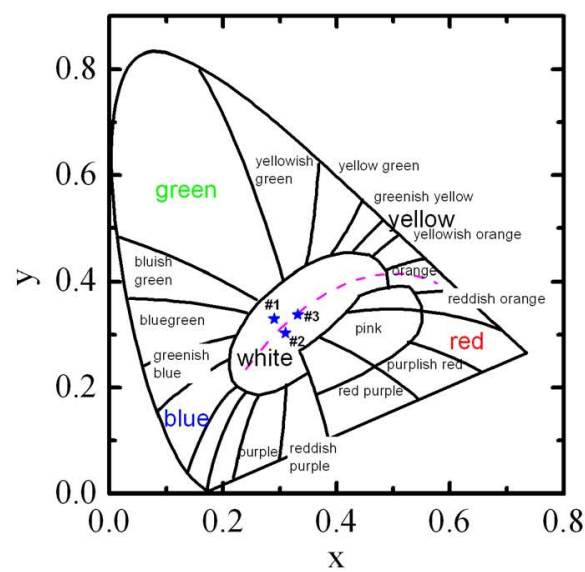

(b)

Fig. 11. (a) EL spectra and (b) associated CIE coordinates of WOLEDs measured under the bias voltage of $10 \mathrm{~V}$.

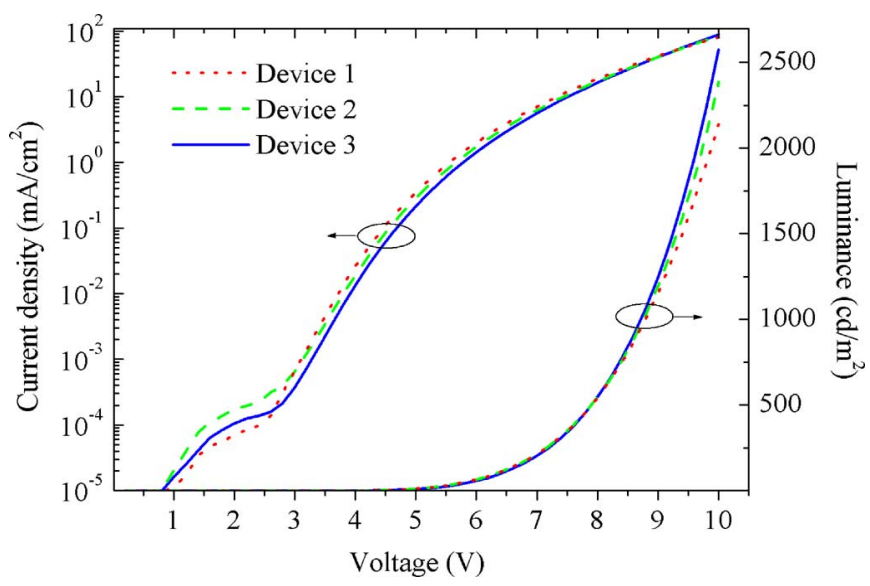

Fig. 12. Current density and luminance as a function of bias voltage for different WOLEDs.

DCM concentration of $3 \mathrm{wt} \%$ ( $1 \mathrm{wt} \%$ in DPVBi $+2 \mathrm{wt} \%$ in $\mathrm{Alq}_{3}$ ). Of those devices, Device 3 still shows the best performance as evident in Fig. 14. On the other hand, the maximum power efficiency (quantum efficiency) of Device 1 and Device 2 drops from 2.58 to $1.24 \mathrm{~lm} / \mathrm{W}$ (from $1.48 \%$ to $0.92 \%$ ) and from 3.16 to $2.15 \mathrm{~m} / \mathrm{W}$ (from $1.96 \%$ to $1.54 \%$ ), respectively, when the DCM concentration increases from $2 \mathrm{wt} \%$ to $3 \mathrm{wt} \%$, a 
TABLE II

PERFORMANCE COMPARISON AMONG WOLEDS

\begin{tabular}{|c|c|c|c|}
\hline & Device 1 & Device 2 & Device 3 \\
\hline Luminance $\left(\mathrm{cd} / \mathrm{m}^{2}\right)$ at $10 \mathrm{~V}$ & 2,140 & 2,386 & 2,575 \\
\hline $\begin{array}{l}\text { Maximum external } \\
\text { quantum efficiency (\%) }\end{array}$ & 1.48 & 1.96 & 1.94 \\
\hline $\begin{array}{l}\text { Maximum power } \\
\text { efficiency }(\mathrm{lm} / \mathrm{W})\end{array}$ & 2.58 & 3.16 & 3.44 \\
\hline CIE $x$ & 0.29 & 0.31 & 0.331 \\
\hline CIE y & 0.33 & 0.303 & 0.337 \\
\hline
\end{tabular}

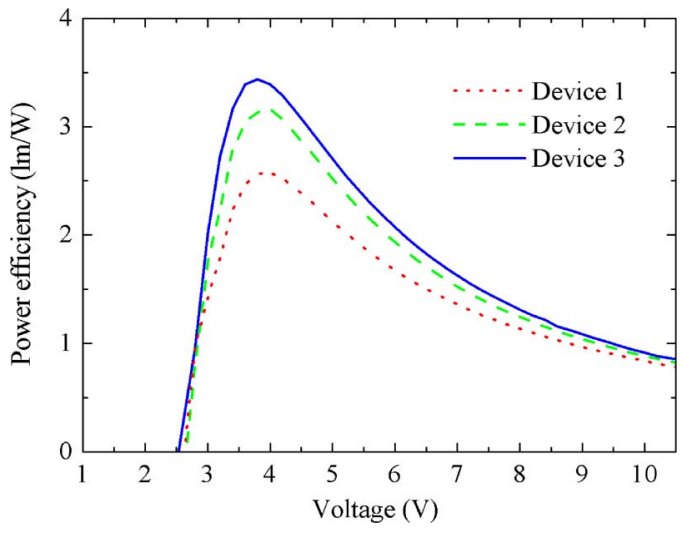

Fig. 13. Power efficiency versus bias voltage for different WOLEDs.

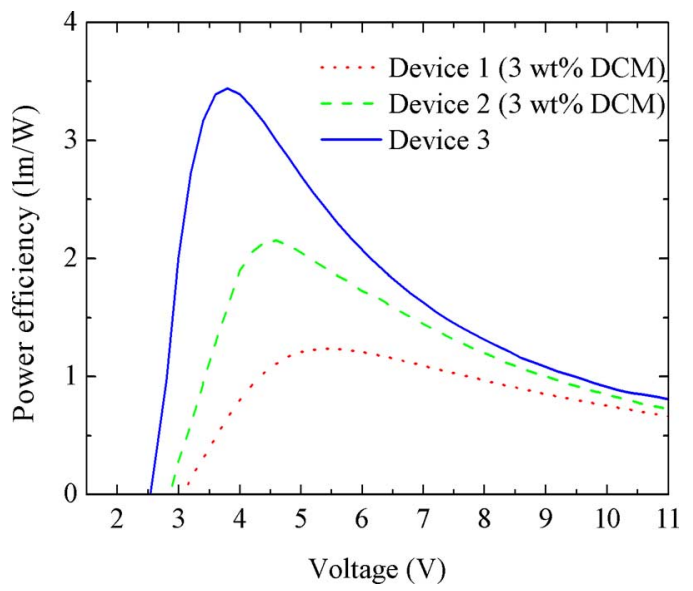

Fig. 14. Power efficiency with respect to bias voltage for different WOLEDs with the overall DCM concentration of $3 \%$ by weight.

direct consequence of self-quenching (singlet-singlet annihilation) [12], [23]. From the simulation result of exciton density distribution presented in Fig. 4, we have found that seed excitons can be confined in the emitting layer. By splitting the doping region into two as done in Device 3, therefore, singlet excitons generated from each layer could be somewhat decoupled or rather confined in the respective layer, singlet-singlet annihilation by which would be suppressed to some extent.

\section{CONCLUSION}

Through a numerical simulation together with experimental analysis, we have first optimized the key design parameters of the WOLED structure (e.g., the doped layer position and thickness and dye concentration). We have then demonstrated through a comparison among different WOLEDs that dual partial dye doping applied to WOLEDs enables performance enhancement as well as chromaticity tuning to the equienergy white point. Such a doping scheme has been shown to broaden the emission bandwidth of a guest dopant (DCM), a desirable peculiarity for white-light generation with two complementary colors. It has also been addressed that the dual partial doping scheme is capable of providing a way of suppressing singlet-singlet annihilation to some extent in the case where a guest dopant needs to be heavily doped.

\section{REFERENCES}

[1] J. Shinar, Organic Light-Emitting Devices: A Survey. College Park, MD: AIP press, 2004.

[2] J. Kalinowski, Organic Light-Emitting Diodes: Principles, Characteristics, and Processes. New York: Marcel Dekker, 2005.

[3] Y. Sun, N. Giebink, H. Kanno, B. Wa, M. E. Thompson, and S. R. Forrest, "Management of singlet and triplet excitons for efficient white organic light-emitting devices," Nature, vol. 440, pp. 908-912, Apr. 2006.

[4] G. Cheng, F. Li, Y. Duan, J. Feng, S. Liu, S. Qiu, D. Lin, Y. Ma, and S. T. Lee, "White organic light-emitting devices using a phosphorescent sensitizer," Appl. Phys. Lett., vol. 82, pp. 4224-4226, Jun. 2003.

[5] H. Kanno, N. C. Giebink, Y. Sun, and S. R. Forrest, "Stacked white organic light-emitting devices based on a combination of fluorescent and phosphorescent emitters," Appl. Phys. Lett., vol. 89, pp. 023503-1-023503-3, Jul. 2006.

[6] G. Cheng, Y. Zhang, Y. Zhao, S. Liu, and Y. Ma, "Improved efficiency for white organic light-emitting devices based on phosphor sensitized fluorescence," Appl. Phys. Lett., vol. 88, pp. 083512-1-083512-3, Feb. 2006.

[7] H. Kanno, Y. Sun, and S. R. Forrest, "White organic light-emitting device based on a compound fluorescent-phosphor-sensitized-fluorescent emission layer," Appl. Phys. Lett., vol. 89, pp. 143516-1-143516-3, Oct. 2006.

[8] G. Schwartz, K. Fehse, M. Pfeiffer, K. Walzer, and K. Leo, "Highly efficient white organic light emitting diodes comprising an interlayer to separate fluorescent and phosphorescent regions," Appl. Phys. Lett., vol. 89, pp. 083509-1-083509-3, Aug. 2006.

[9] G. Li and J. Shinar, "Combinatorial fabrication and studies of bright white organic light-emitting devices based on emission from rubrenedoped 4, 4'-bis(2, 2'-diphenylvinyl)-1, 1'-biphenyl," Appl. Phys. Lett., vol. 83, pp. 5359-5361, Dec. 2003.

[10] K. O. Cheon and J. Shinar, "Bright white small molecular organic light-emitting devices based on a red-emitting guest-host layer and blue-emitting 4, 4'-bis(2, 2'-diphenylvinyl)-1, 1'-biphenyl," Appl. Phys. Lett., vol. 81, pp. 1738-1740, Aug. 2002.

[11] R. S. Deshpande, V. Bulović, and S. R. Forrest, "White-light-emitting organic electroluminescent devices based on interlayer sequential energy transfer," Appl. Phys. Lett., vol. 75, pp. 888-890, Aug. 1999.

[12] S.-H. Yang, M.-H. Liu, and Y.-K. Su, "Stable and highly bright with organic light-emitting diode based on $4,4^{\prime}, 4^{\prime \prime}$-tris(n-3-methylphenyl-nphenyl-amino)-triphenylamine," J. Appl. Phys., vol. 100, pp. 0831111-083111-4, Oct. 2006.

[13] H. Choukri, A. Fischer, S. Forget, S. Chénais, M.-C. Castex, D. Adés, A. Siove, and B. Geffroy, "White organic light-emitting diodes with fine chromaticity tuning via ultrathin layer position shifting," Appl. Phys. Lett., vol. 89, pp. 183513-1-183513-3, Nov. 2006. 
[14] B. Ruhstaller, T. Beierlein, H. Riel, S. Karg, J. C. Scott, and W. Riess, "Simulating electronic and optical processes in multilayer organic light-emitting devices," IEEE J. Sel. Top. Quantum Electron., vol. 9, pp. 723-731, May/Jun. 2003.

[15] J. Staudigel, M. Stößel, F. Steuber, and J. Simmerer, "A quantitative numerical model of multilayer vapour-deposited organic light emitting diodes," J. Appl. Phys., vol. 86, pp. 3895-3910, Oct. 1999.

[16] B. Ruhstaller, S. A. Carter, S. Barth, H. Riel, W. Riess, and J. C. Scott, "Transient and steady-state behaviour of space charges in multilayer organic light-emitting diodes," J. Appl. Phys., vol. 89, pp. 4575-4586, Apr. 2001

[17] G. G. Malliaras and J. C. Scott, "The roles of injection and mobility in organic light emitting diodes," J. Appl. Phys., vol. 83, pp. 5399-5403, May 1998.

[18] G. G. Malliaras and J. C. Scott, "Numerical simulations of the electrical characteristics and the efficiencies of single-layer organic light emitting diodes," J. Appl. Phys., vol. 85, pp. 7426-7432, May 1999.

[19] P. W. M. Blom, M. J. M. de Jong, and S. Breedijk, "Temperature dependent electron-hole recombination in polymer light-emitting diodes," Appl. Phys. Lett., vol. 71, pp. 930-932, Aug. 1997.

[20] J. M. Lupton and I. D. W. Samuel, "Temperature-dependent single carrier device model for polymeric light emitting diodes," J. Phys. D: Appl. Phys., vol. 32, pp. 2973-2984, Dec. 1999.

[21] J. W. Park and Y. Kawakami, "Temperature-Dependent dynamic behaviors of organic light-emitting diode," J Display Technol, vol. 2, no. 4, pp. 333-340, Dec. 2006.

[22] C.-C. Lee, M.-Y. Chang, P.-T. Huang, Y. C. Chen, Y. Chang, and S.-W Liu, "Electrical and optical simulation of organic light-emitting devices with fluorescent dopant in the emitting layer," J. Appl. Phys., vol. 101, pp. 114501-1-114501-11, Jun. 2007.

[23] H. Nakanotani, H. Sasabe, and C. Adachi, "Singlet-singlet and singletheat annihilations in fluorescence-based organic light-emitting diodes under steady-state high current density," Appl. Phys. Lett., vol. 86, pp. 213506-1-213506-3, May 2005.

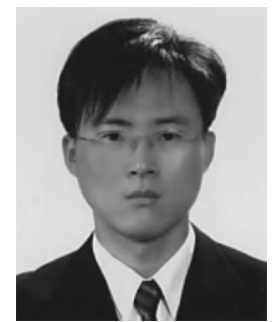

Jongwoon Park received the B.S. degree in radio engineering from Kyunghee University, Suwon, Korea, in 1999, the M.S. degree from the University of Victoria, Victoria, BC, Canada, in 2001, and the Ph.D. degree in electrical engineering from McMaster University, ON, Canada, in 2004.

He joined the Department of Electronic Science and Engineering at Kyoto University, Kyoto, Japan, as a postdoctoral fellow in 2005. Since 2007, he has been working for Korea Institute of Industrial Technology (KITECH), Gwangju, Korea, as a senior researcher. His research interests are organic light-emitting diodes (OLEDs), GaN-based light-emitting devices, fiber-optic communication systems, and numerical modeling and simulation of semiconductor optoelectronic devices.

Naotoshi Suganuma received the B.Eng, M.Eng., and D.Eng. degrees in Functional Polymer Science from Shinshu University, Ueda, Japan, in 1998, 2000, and 2003, respectively.

$\mathrm{He}$ is currently working as a postdoctoral fellow in International Innovation Center at Kyoto University, Kyoto, Japan. His research interests are driving physics and fabrication technology of organic light-emitting devices and organic transistors.

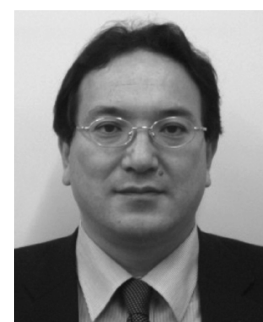

Yoichi Kawakami was born in Ehime, Japan, in 1961. He received the B.Sc., M.Sc., and D.Sc degrees in electrical engineering from Osaka University in 1984, 1986 and 1989, respectively.

After graduation, he joined at Department of Electrical Engineering, Kyoto University as a research associate, working on II-VI widegap semiconductors. Since 1991 to 1992 , he had been a visiting research fellow at Department of Physics, Heriot-Watt University in UK. In 1997, he became an associate professor at Department of Electronic Science and Engineering, Kyoto University. His current research is focused on spatial and temporal luminescence spectroscopy of excitons in GaN-based low-dimensional semiconductors, as well as on the application of solid-state lighting using white light emitting diodes. 